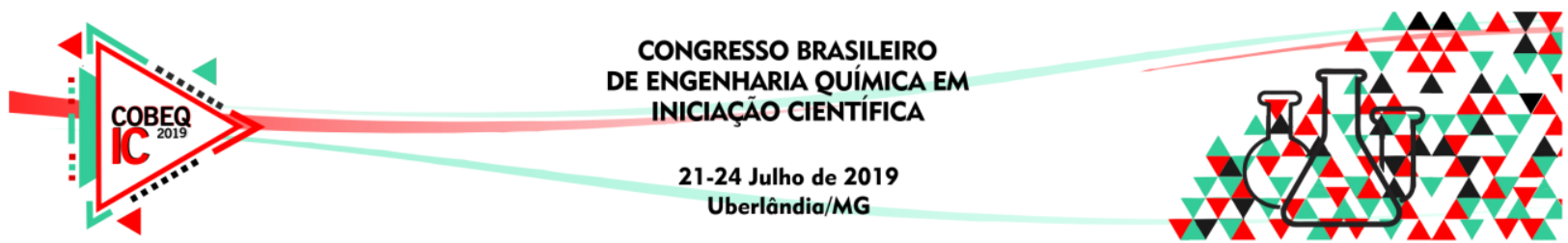

\title{
REUTILIZAÇÃO DE FIBRAS DE AÇAÍ (Euterpe Oleracea) PARA A PRODUÇÃO DE PLACAS CIMENTÍCIAS
}

\author{
M. S. FAVACHO ${ }^{1}$, M. D. C. ALMEIDA ${ }^{1}$ \\ ${ }^{1}$ Universidade do Estado do Amapá, Colegiado de Engenharia Química \\ E-mail para contato: matheusfavachomacapa@gmail.br
}

\begin{abstract}
RESUMO - O presente trabalho busca viabilizar a utilização de fibras vegetais, oriundas de resíduos da cadeia produtiva do açaí, e papel para fabricação de um material compósito com cimento como material alternativo para o setor de construção civil. Desta forma, busca-se uma alternativa no reaproveitamento dos resíduos para a produção de um novo compósito para o mercado. Para tanto, produziu-se corpos-deprova com teor de fibra de $5 \%$ e $1 \%$ de papel e utilizaram-se ensaios físico-mecânicos (ensaios de resistência à tração na flexão, absorção de água, densidade aparente e variação dimensional) para avaliação de desempenho do material final. Através do ensaio mecânico de flexão, as placas cimentícias obtiveram resultados mínimos de 2,07 MPa, o qual é compatível com a NBR 15498/2016 para aplicação como revestimento não estrutural de ambientes externos. A adição de fibras de açaí contribuiu no ganho de resistência a flexão, indicando sua viabilidade para a produção das placas cimentícias conforme a NBR 15498/2016.
\end{abstract}

PALAVRAS CHAVE: Compósito; Material; Resíduos.

\section{INTRODUÇÃO}

Dentre os diversos resíduos que apresentam grande potencialidade para produção de novos materiais está o resíduo da cadeia produtiva do açaí, produto consumido como hábito alimentar quase que diariamente por grande parte da população do Amapá e do Pará. Este resíduo, rico em fibras e que possui um caroço lenhoso, possui várias aplicações, como a compostagem e reutilização como briquete para queima em fornos cerâmicos Rocha (2013). Contudo, por vezes, este resíduo produzido pelos batedores de açaí é despejado nas imediações de ruas, avenidas e córregos gerando um impacto ambiental. Tal desperdício, descrito por Costa, Monteiro e Pinheiro (2017), resulta em uma grande quantidade de caroços, que, muitas vezes, são destinados ao lixão municipal gerando uma grande perda de potencialidade que o caroço de açaí possui.

Com isso, placas cimentícias com a incorporação de fibras de açaí utilizando em sua composição um teor de 5\% de fibra, vêm com uma inovação por utilizar resíduos da extração de fibra do carroço de açaí e aplicar em um material de construção. Estas mudanças modernizam as práticas no canteiro de obra, com menos desperdícios e mais objetividade, tornando a atividade mais dinâmica e com resultados muito mais satisfatórios para todos os envolvidos.

Existem estudos que visam à utilização de fibras lignocelulósicas como reforço de materiais compósitos (Santos et al., 2011; Silva et al., 2009). Para Goulart, Brandes, Souza, 


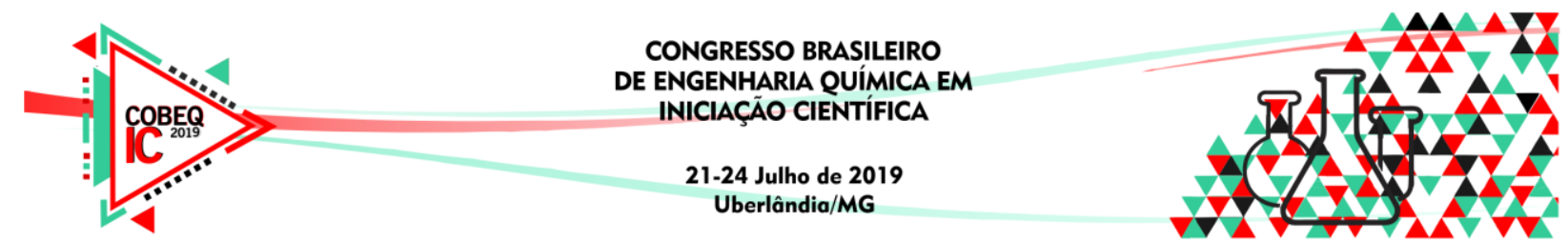

Carminatti, Mikowski, Michél, Oliveira e Recouvreux (2016), os resultados obtidos mostraram que as fibras de açaí exibem excelente potencial estrutural e representam uma alternativa viável para a produção de um material com grande valor agregado. Segundo Junior (2007), as fibras de açaí, incrementam de forma satisfatória a resistência à tração de compósito fibrocimentícios (aproximadamente $13 \%$ em média), com a melhoria das propriedades mecânicas.

Portanto, as placas cimentícias com a incorporação de fibras de açaí utilizando em sua composição um teor de $5 \%$ de fibra, vêm com uma inovação por utilizar resíduos da extração de fibra do carroço de açaí e aplicar em um material de construção. Estas mudanças modernizam as práticas no canteiro de obra, com menos desperdícios e mais objetividade, tornando a atividade mais dinâmica e com resultados muito mais satisfatórios para todos os envolvidos.

\section{MATERIAIS E MÉTODOS}

\subsection{Formulação e conformação dos Corpos de Prova}

A formulação utilizada para a produção do traço foi a seguinte proporção: para cada $1 \mathrm{~kg}$ de cimento Portland CP-II-Z-32, utilizaram-se $2 \mathrm{~kg}$ de areia com grãos de 1,16 mm, 0,15 $\mathrm{kg}$ (teor de $5 \%$ em relação a massa total) de fibra de açaí in natura, 0,020 L de aditivo plastificante baseado em Breu saponificado $\left(\mathrm{SIKA}^{\circledR}\right)$ e $0,030 \mathrm{~kg}$ (teor de $1 \%$ ) de papel reciclado coletado de lixeiras da universidade. O método para produção da placa cimentícia baseou-se na NBR 15498:2016, que apresenta requisitos e estabelece métodos de ensaio e as condições de recepção das placas planas cimentícias reforçadas com fibras, fios, filamentos ou telas.

O preparo da mistura para conformação dos corpos de prova (CPs) foi realizada em uma forma metálica, adicionando-se os materiais conforme a composição descrita no parágrafo anterior e 0,5 litros de água, e homogeneizando-se com auxílio de uma espátula. A mistura foi então vertida em 3 moldes de poliestireno expandido (CP 01, CP 02, CP 03), cujas dimensões eram $0,03 \mathrm{~m} \times 0,15 \mathrm{~m} \times 0,15 \mathrm{~m}$. O CPs foram deixados para cura em temperatura ambiente por aproximadamente 14 dias.

\section{RESULTADOS}

A figura 1 apresenta os valores de adsorção de água dos corpos de prova das placas para a composição adotada. Observa-se que os valores médios de absorção dos corpos de prova foram de $16,59 \% \pm 0,89$. 


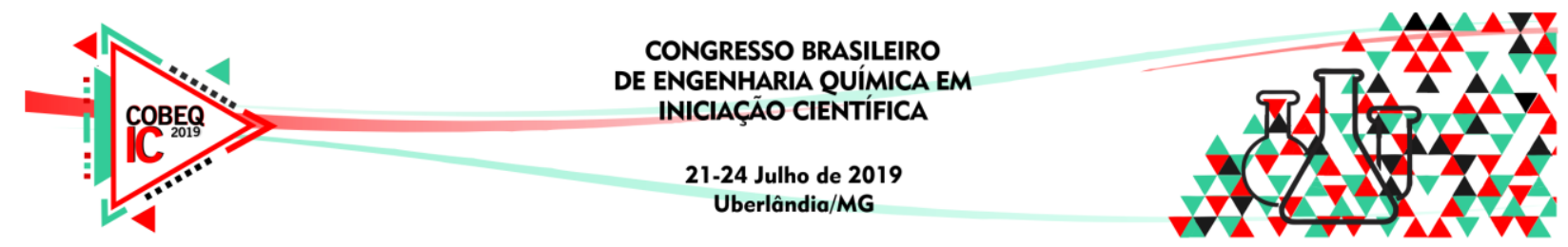

Figura 1 - Comparativo Percentual de Absorção de Água.

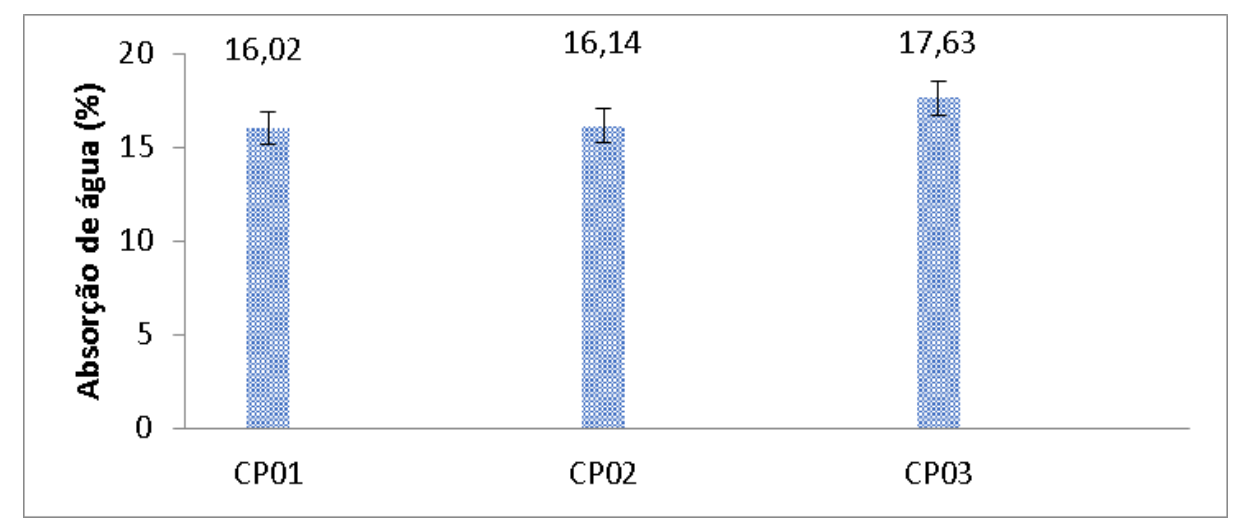

Anjos; Gravami; Barbosa (2003) desenvolveram placas cimentícias com uso de polpa celulósica de bambu. Neste trabalho obtiveram resultados muito superiores de absorção de água, ou seja, tem uma resistência minorada com a exposição a umidade. Com teor de absorção dos CP 01, CP 02 e CP 03 encontrados no desenvolvimento deste trabalho, indicando possivelmente, que o compósito obtido neste estudo comparado ao trabalho de Anjos; Gravami; Barbosa (2003), possui uma resistência superior quanto à exposição a umidade.

Para Aggarwal (1995), a retenção de umidade por partículas contidas no reforço de compósitos de matriz cimentícia são responsáveis pela lixiviação de compostos solúveis em água, o que diminui a resistência do cimento, bem como, degrada a fibra vegetal devido à decomposição de ligninas de baixo peso molecular e hemiceluloses. A retenção de umidade por superfícies cimentícias é uma das principais causas do aparecimento de mofos e da diminuição de adesão ao revestimento (tinta).

Para o ensaio de densidade aparente, foram utilizados os corpos de prova (CP 01, CP 02 e CP 03) $0,15 \mathrm{~m} \times 0,15 \mathrm{~m} \times 0,03 \mathrm{~m}$. As informações dos resultados encontrados de densidade aparente podem ser encontradas na Tabela 1.

Tabela 1 - Densidade Aparente dos Corpos de Prova

\begin{tabular}{|c|c|c|c|c|}
\hline Grandezas & CP 01 & CP 02 & CP 03 & Desvio \\
\hline Massa Final $(\mathrm{g})$ & 1295,04 & 1258,65 & 1142,65 & \multirow{2}{*}{7,12} \\
\cline { 1 - 3 } Volume $(\mathrm{ml})$ & 18,68 & 21,36 & 15,75 & \\
\cline { 1 - 3 } $\begin{array}{c}\text { Densidade Aparente } \\
\left(\mathrm{g} / \mathrm{cm}^{3}\right)\end{array}$ & 69,33 & 58,93 & 72,55 & \\
\hline
\end{tabular}

A variação dimensional torna-se fundamental para as placas cimentícias com fins de vedação, visto que estes sistemas em geral ficam fixados como em estruturas metálicas, por exemplo, e quando sujeitas a exposição a umidade podem ter suas dimensões alteradas o que afeta a integridade do material e do sistema. Também contribui para a variação dimensional é a precisão do paquímetro, pelo fato do erro instrumental e o posicionamento no material na hora da medição. Para ter o detalhamento dessa variação, teve-se que aumentar o número de casas decimais. 


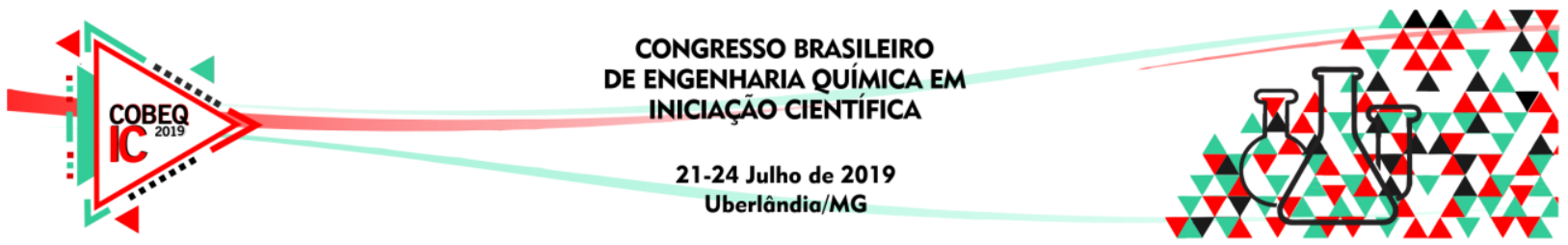

Tabela 2 - Variação Dimensional dos Corpos de Prova

\begin{tabular}{|c|c|c|c|c|c|}
\hline Grandezas & CP 01 & CP 02 & CP 03 & Média & Desvio \\
\hline $\begin{array}{c}\text { Comprimento } \\
\text { Inicial (cm) }\end{array}$ & 15,30 & 15,05 & 15,23 & 15,19 & \multirow{2}{*}{0,37} \\
\cline { 1 - 4 } $\begin{array}{c}\text { Comprimento } \\
\text { Final (cm) }\end{array}$ & 15,31 & 14,98 & 15,30 & 15,19 & \\
\cline { 1 - 3 } $\begin{array}{c}\text { Variação } \\
\text { Dimensional (cm) }\end{array}$ & $-0,06$ & 0,46 & $-0,45$ & $-0,02$ & \\
\hline
\end{tabular}

A NBR 15498/2016 não faz referência explicita sobre quais valores seriam os ideais para placas cimentícias. A redução e a expansão relacionam-se com o paquímetro medido em posições diferentes com uma diferença instrumental. Também a retração verificada pelo uso do líquido fino deve-se a característica do resíduo, que mesmo com pouco percentual de partículas do resíduo, ainda desempenha um papel de absorção da umidade externa importante, além de nos casos do uso de aditivos incorporadores de ar, essa variação é compensada pelas bolhas de ar internas geradas pelo seu uso (VAN GERVEN et al, 2007).

Quanto ao ensaio realizado de tensão de ruptura à flexão, a resistência média obtida foi de 2,35 MPa $\pm 0,37$. Segundo a norma NBR 15498/2016 a resistência de 2,35 MPa fica classificada como placa de fibrocimento classe A de categoria 1 como demostra a tabela 3 com devidas informações da placa de fibrocimento para aplicações em áreas externas. A Figura 02 apresenta o gráfico de histograma da tensão de ruptura à flexão dos corpos de prova produzidos com os respectivos desvios.

Tabela 3 - Tabela de resistência a flexão NBR 15498:2016

\begin{tabular}{|c|c|c|}
\hline \multicolumn{3}{|c|}{ Requisitos de resistência à flexão } \\
\hline \multicolumn{3}{|c|}{$\begin{array}{c}\text { Norma NBR 15498:2016 - Placa de fibrocimento sem amianto - Requisitos e métodos de } \\
\text { ensaio }\end{array}$} \\
\hline Categoria & $\begin{array}{c}\text { Placas da classe A Aplicações } \\
\text { Externas (MPa) } \\
\end{array}$ & $\begin{array}{c}\text { Placas da classe B } \\
\text { Aplicações Internas (MPa) }\end{array}$ \\
\hline 1 & 0 a 4 & 4 \\
\hline
\end{tabular}

Karade (2010), em estudo sobre compósitos cimentícios reforçados com resíduos lignocelulósicos evidenciou que em trabalhos que utilizaram como reforço cascas de arroz, os valores obtidos para resistência à ruptura a flexão foram similares aos encontrados neste trabalho.

De maneira geral, obtiveram-se chapas com potencialidade para aplicação não estrutural na indústria da construção civil. Azevedo (2018) em seu trabalho para a produção de placas cimentícias com uso de lodo da indústria de papel obteve resistências à tensão de ruptura abaixo de $1,3 \mathrm{MPa}$. 


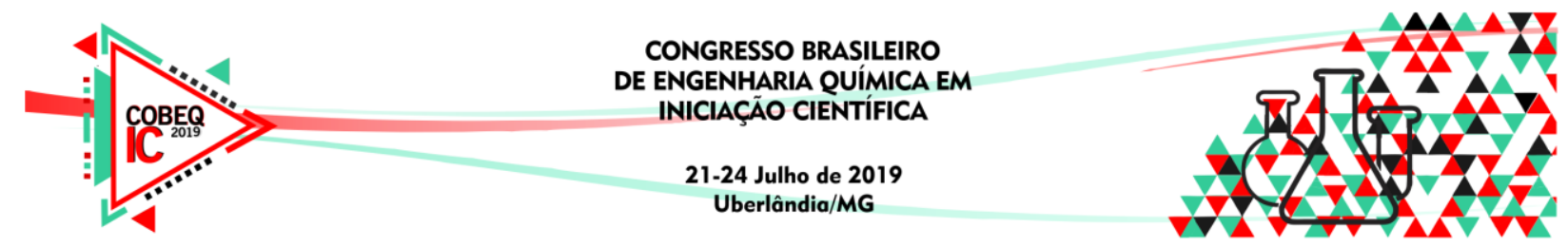

Figura 2 - Valores de Tensão de Ruptura à Flexão dos Corpos de Prova

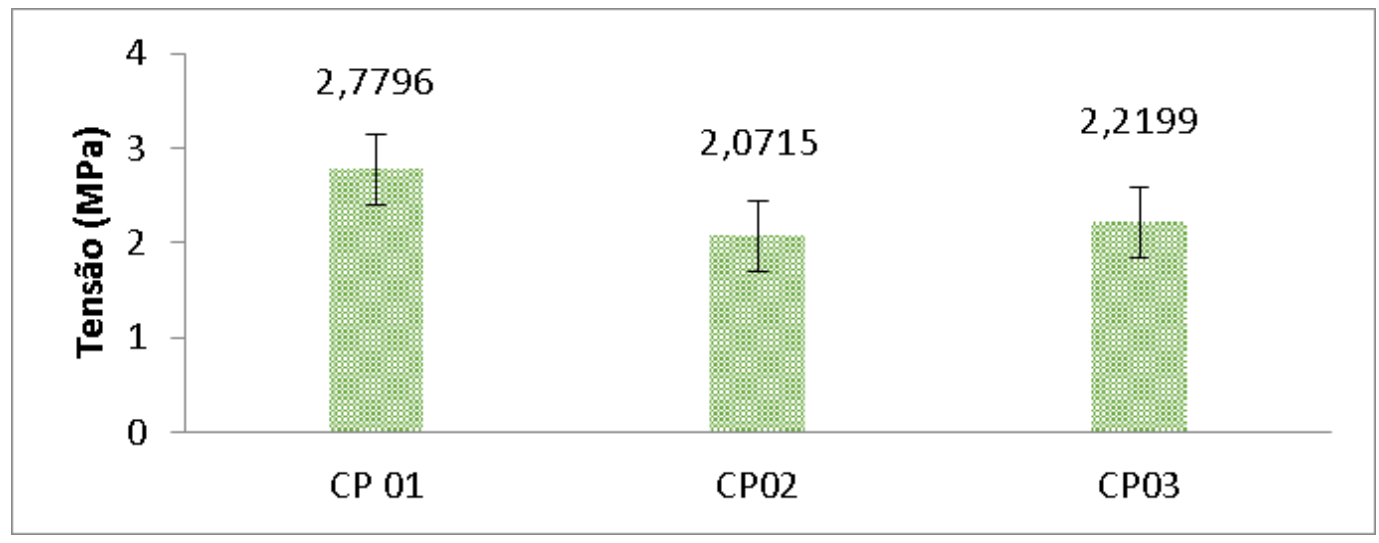

O resultado de resistência a flexão demostrado pela fica figura 2 leva em consideração a importância da quantidade de fibra utilizada no compósito, bem como, a utilização de porzolanas com intuito de melhorar características mecânicas do material obtido.

\section{CONSIDERAÇÕES FINAIS}

Podemos considerar que devido o teor de $5 \%$ de fibra de açaí não obteve a resistência de flexão desejada comparada a outros trabalhos da literatura, contudo, considerando a Norma NBR 15498/2016, a placa cimentícias obtida encontra uso com revestimento não estrutural, desta forma, sendo viável. No entanto, há a necessidade de avaliar maiores teores de fibra no material com o objetivo de obter-se um produto com uma resistência superior à determinada no ensaio. Além disso, há a necessidade de otimização do processo de homogeneização das fibras com a matriz cimentícia para promover uma maior resistência mecânica do material.

\section{LISTA DE NOTAÇÕES E SÍMBOLOS UTILIZADOS}

$\mathrm{cm}-$ Centímetro $=10^{-2}$ Metro

CP-II-Z-32 - Cimento Portland composto com pozolana

CP - Corpo de Prova

$\mathrm{kg}$ - Kilograma

$\mathrm{MPa}-$ Mega Pascal $=10^{6}$ Pascal

$\mathrm{m}$ - Metro

$\mathrm{mm}-$ Milímetro $=10^{-3}$ Metro

$\%$ - Porcentagem

(B) - Registrado

\section{REFERÊNCIAS}

AGGARWAL, L. K. Bagasse-Reinforced Cement Composites. Cement and concrete composite. v.17, 1995, p. 107- 112

ANJOS, M. A. S.; GRAVAMI, K.; BARBOSA, N. Compósitos à base de cimento reforçados com polpa celulósica de bambu. Revista Brasileira de Engenharia Agrícola e Ambiental, v.7, n.2, p.339-345, 2003 


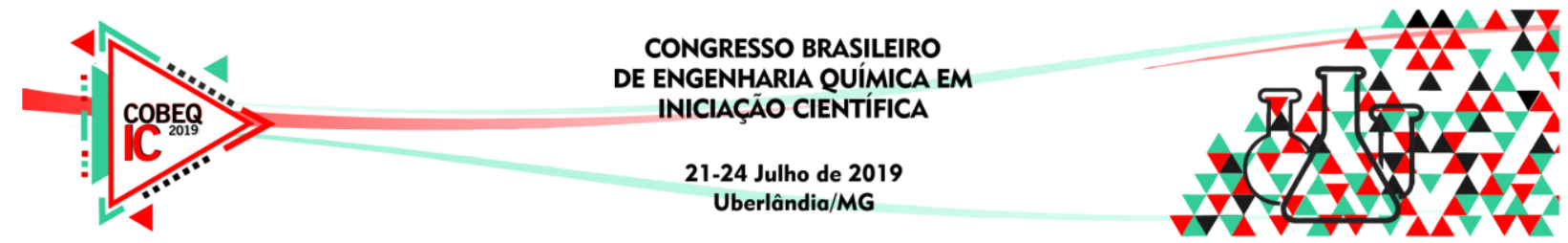

AZEVEDO, A.R.G. Desenvolvimento de placas cimentícias reforçadas com lodo primária da indústria de papel de celulose. Tese (doutorado). Universidade Estadual do Norte Fluminense. Programa de Pós-Graduação em Engenharia Civil. Campos dos Goytacases, 2018. 168 p.

ASSOCIAÇÃO BRASILEIRA DE NORMAS TÉCNICAS (ABNT), NBR 15498: Placa de fibrocimento sem amianto. Rio de Janeiro, 2016.

KARADE, S. R. Cemente-bonded composites lignocellulosic wastes. Construction and building materials. v.24, 2010, p. 1323-1330.

GOULART, L.; BRANDES, R.; SOUZA, L.; CARMINATTI, C. A.; MIKOWSKI, A.; MICHÉL, M. D.; OLIVEIRA, E. M.; RECOUVREUX, D. O. S. Preparação $e$ caracterização do biocompósito amido termoplástico/fibras de açaí. $22^{\circ}$ CBECiMat Congresso Brasileiro de Engenharia e Ciência dos Materiais 06 a 10 de novembro de 2016, Natal, RN, Brasil.

JÚNIOR, U. M. L. Fibras da semente do açaizeiro (Euterpe Oleracea Mart.): avaliação quanto ao uso como reforço de compósitos fibrocimentícios. 2007.145 f. Dissertação (Mestrado em Engenharia e Tecnologia de Materiais) - Pontifícia Universidade Católica do Rio Grande do Sul, Porto Alegre, 2007.

MONTEIRO, L. H. O.; COSTA, R. O. S.; PINHEIRO, P. F. V. Resíduos produzidos pelos processadores de açaí na região metropolitana de belém e avaliação do seu potencial energético. IX Simpósio Brasileiro de Engenharia Ambiental, XV Encontro Nacional de Estudantes de Engenharia Ambiental e III Fórum Latino Americano de Engenharia e Sustentabilidade. 2017. Belo Horizonte. http://pdf.blucher.com.br.s3-sa-east 1.amazonaws.com/engineeringproceedings/xveneeamb/153.pdf. Acesso em: 12 de dezembro de 2016.

ROCHA, A. L. Alternativas para o abastecimento energético dos fornos de indústrias cerâmicas localizadas no município de São Miguel do Guamá-PA. Tese (mestrado). Universidade Federal do Pará. Programa de Pós-Graduação em Engenharia Civil, Belém, 2013.

SANTOS, A. W. L.; LIMA, I.V.; SOUZA, I. B.F.; FRANCISCO, J.D.; FRANCO, P.A.; OLIVEIRA. P.F.; SILVA, S.C.R.; VALÉRIO, T.N.; SILVA, A.V. Piso Produzido a partir de Fibras Vegetais. E- xacta, Belo Horizonte, v. 4, n. 2 - Edição Especial Interdisciplinaridade. p. 59 - 64. Editora UniBH. 2011.

SILVA, R.; HARAGUCHI, S.K.; MUNIZ, E. C.; RUBIRA, A.F.; Aplicações de fibras lignocelulósicas na química de polímeros e em compósitos. Quim Nova, v.32, p.661, 2009.

VAN GERVEN, T. Effects of Carbonation and Leaching on Porosity in Cement-Bound Waste. Waste Management, v. 27, n. 7, p. 977-985, 2007. 